\title{
A Search for Variable Interstellar Magnetic Fields and Neutron Star Precession in Four Years of Polarization Position Angle Measurements on 98 Pulsars
}

\author{
J. M. Weisberg, J. J. Morgan, J. T. Despotes, \& J. E. Everett \\ Department of Physics \& Astronomy, Carleton College, Northfield, $M N$ \\ 55057 USA \\ J. M. Cordes \\ Department of Astronomy \& $N A I C$, Cornell University, Ithaca, NY \\ 14853 USA
}

\begin{abstract}
We have studied the temporal behavior of linearly polarized position angle in a sample of 98 pulsars observed from Arecibo over a four-year period in a search for free precession and changes in interstellar magnetic fields along pulsar- earth lines of sight. We set upper limits on changes in position angle.
\end{abstract}

\section{Introduction and Observational Analyses}

In order to search for free precession and changes in interstellar Faraday rotation, we determined full Stokes parameters on all sufficiently strong pulsars in our sample of 98 pulsars observed from Arecibo at $21 \mathrm{~cm}$ from 1989 through 1993 (Weisberg et al 1999). All pulsars' position angles were referenced either to $\mathrm{B} 1929+10$ or $\mathrm{B} 0540+23$, which were found to have strong and constant linear polarization. (See Figure 1.)

\section{Free Precession and Interstellar Magnetic Field Variations}

If the mass of an isolated rotating body is distributed asymmetrically with respect to its spin axis, the body will undergo "free precession" (Sedrakian et al 1999). For an animation of this process, see Everett \& Weisberg (1997). Probably the clearest observational evidence for free precession would be temporal changes in polarized position angle of emission, $\Delta \psi$.

The interstellar Faraday rotation measure $(R M)$, in combination with dispersion measure $(D M)$, provides a tool for measurement of the magnetic field along a pulsar-Earth path. There are only a few reports of changing Faraday rotation for sources other than the young Crab and Vela Pulsars (e.g., Han, Manchester, \& Qiao 1999). The fractional change in parallel component of magnetic field, $\Delta\left\langle B_{\| \mid}\right\rangle /\left\langle B_{\|}\right\rangle$,

$$
\frac{\Delta\left\langle B_{\|}\right\rangle}{\left\langle B_{||}\right\rangle}=\frac{\Delta[R M]}{R M}=\frac{\Delta[\psi(\lambda)]}{R M \lambda^{2}},
$$



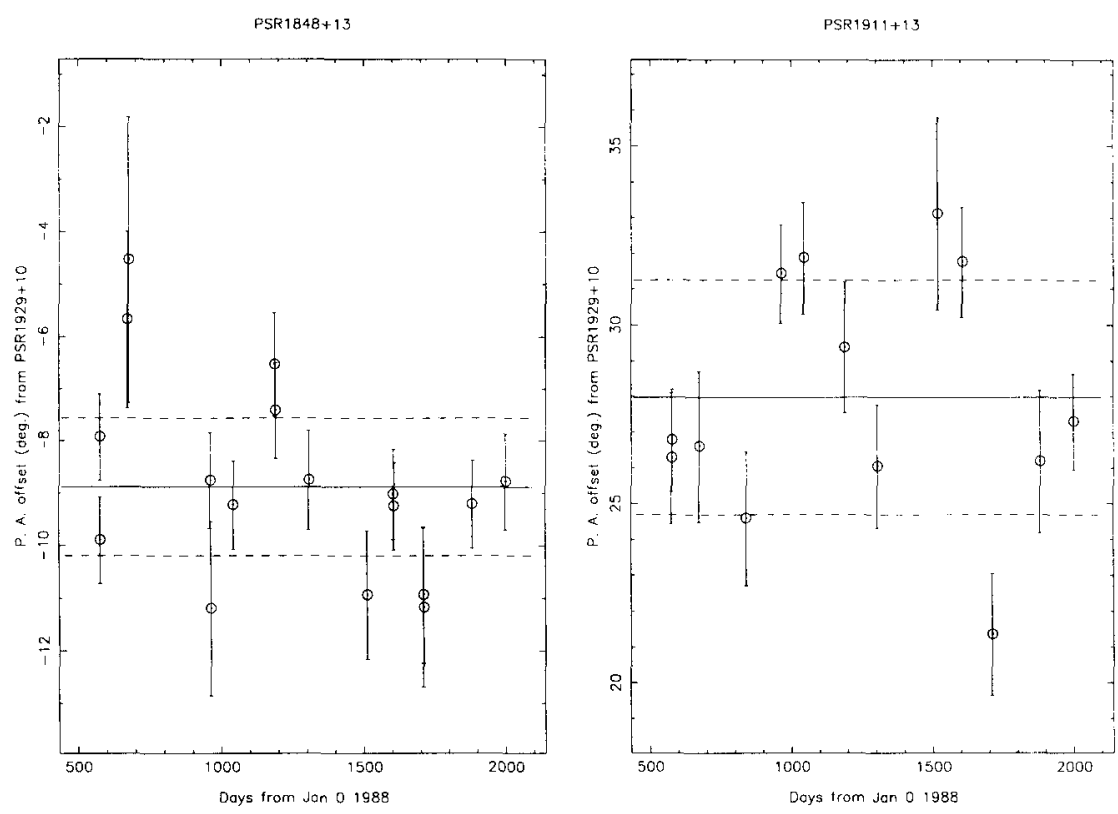

Figure 1. Position angles of two pulsars versus time. The solid line is the weighted mean $\psi$; each dotted line represents a $1-\sigma$ offset.

where $\Delta \psi(\lambda)$ is the measured change in position angle at a particular wavelength $\lambda$. We can then insert our observed $\Delta \psi$ (or limits thereon) into Equation (1) to study or limit interstellar magnetic field variations.

\section{Results}

We searched for position angle variations in the 84 pulsars for which we had adequate data to analyze. If a change were seen, it could be explained as the result of free precession or variable interstellar magnetic fields. We detected no evidence for significant variations in position angle.

\section{References}

Everett, J. E., \& Weisberg, J. M. 1997, "The Free Precession of an Oblate Star," Mathematica Notebook on the MathSource Library, at URL www.mathsource.com/cgi-bin/MathSource/Applications/Astronomy/0208-572

Han, J. L., Manchester, R. N., \& Qiao, G. J. 1999, MNRAS, 306, 371

Sedrakian, A., Wasserman, I., \& Cordes, J. M. 1999, ApJ, 524, 341

Weisberg, J. M., Cordes, J. M., Lundgren, S. C., Dawson, B. R., Despotes, J. T., Morgan, J. J., Weitz, K. A., Zink, E. C., \& Backer, D. C. 1999, ApJS, 121,171 